\title{
THE EFFECTS OF WARM-UP DURATION ON CYCLING TIME TRIAL PERFORMANCE IN TRAINED CYCLISTS
}

\author{
Jennifer A. Bunn, ${ }^{1, A, B, C, D}$ L. Chris Eschbach, ${ }^{2, A, B, C}$ Meir Magal,, ${ }^{3, B, B, C}$ Elizabeth K. Wells, \\ ${ }^{1}$ Department of Physical Therapy, Campbell University, Buies Creek, NC, USA \\ ${ }^{2}$ Valencell Inc. Raleigh, NC, USA \\ ${ }^{3}$ Mathematics and Sciences Division, North Carolina Wesleyan College, Rocky Mount, NC, USA

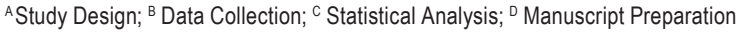 \\ Address for corpespondence: \\ Jennifer Bunn \\ Campbell University, Department of Physical Therapy, \\ 4250 US 421 South, Lillington, NC 27546; 910-893-1361 \\ Email:bunnj@campbell.edu
}

\begin{abstract}
Ahstract The purpose of this study was to assess the effects of three different warm-up condi-tions on a $5 \mathrm{~K}$ cycling time trial (TT). Sixteen trained cyclists completed the study. At the first testing session, participants completed a maximal graded exercise test to assess maximal oxygen consumption ( $\mathrm{VO}_{2} \mathrm{max}$ ) and a familiarization of the TT. At three subse-quent visits, the participants completed the TT after no warm up, short warm-up of three minutes at $60 \% \mathrm{VO}_{2} \max$, or long warm-up of ten minutes at $60 \% \mathrm{VO}_{2} \max$. The warm-up was assigned in randomized order. $\mathrm{VO}_{2}$, heart rate $(\mathrm{HR})$, lactate, power, and speed were assessed after the warm-up, $1 \mathrm{~K}$, and completion of the $5 \mathrm{~K}$ TT. There was no dif-ference between type of warm-up for time, power, cadence, speed, $\mathrm{VO}_{2}, \mathrm{HR}$, or lactate levels at the end of the TT. There was no significant difference between type of warm-up for time, $\mathrm{VO}_{2}$ or $\mathrm{HR}$ at the end of the $1 \mathrm{~K}$ split. Warm-up length was not impactful on $5 \mathrm{~K}$ TT performance or during the first $\mathrm{km}$ of the TT in trained cyclists. These results con-flict with previous evidence indicating that a warm-up in endurance events primarily improved $\mathrm{VO}_{2}$ kinetics at the onset of the exercise.
\end{abstract}

Key Worlds Maximal oxygen consumption, endurance training, lactate, oxygen kinetics, aerobic

\section{Introduction}

Completing a warm-up before anaerobic and aerobic exercise may prepare vari-ous physiological mechanisms that athletic performance relies upon including: blood flow, oxygen kinetics and metabolic activity (Hajoglou et al., 2005; Johnson, Gregson, Mills, Gonzalez, Sharpe, 2014; Tomaras, Maclntosh, 2011). Warm-up methods have two broad classifications: passive and active warm-ups (Bishop, 2003a, 2003b). This study focuses on active warmups which consist of using exercise (e.g. jogging, cycling, and swimming) that produces the same effect as a passive warm-up (Bishop, 2003a, 2003b). There are numerous studies that have determined the benefits of a passive or active warm-up for short duration, high intensity and resistance exercise (Bishop, 2003a; Gray, Nimmo, 2001); however, there are conflicting studies that contest whether a warm-up will improve (Bishop, 2003b), hinder (Andzel, 
Busuttil, 1982; Gregson, Drust, Batterham, Cable, 2002), or have no effect (Andzel, 1978; Andzel, Busuttil, 1982; Andzel, Gutin, 1976; Grodjinovsky, Magel, 1970) on long-term endurance exercise. The amount of time between the warm-up and performance is also important. A transition time of less than five minutes appears to be most effective (Andzel, 1978, 1982; Andzel, Gutin, 1976; Genovely, Stamford, 1982; Grodjinovsky, Magel, 1970) as the athlete is able to recover from the warm-up bout, and will likely begin the exercise with an elevat-ed oxygen consumption $\left(\mathrm{VO}_{2}\right)$ and muscle temperature and therefore be more prepared for the performance.

Studies reporting improvement in endurance performance related to warm up at-tribute the benefit to an increase in VO 2 (Andzel, 1978; Andzel, Gutin, 1976; Genovely, Stamford, 1982; Grodjinovsky, Magel, 1970; Martin, Robinson, Wiegman, Aulick, 1975), an increase in heart rate (HR) (Andzel, 1978; Andzel, Gutin, 1976; Martin et al., 1975), a decrease in lactate accumulation (Gerbino, Ward, Whipp, 1996; Kozlowski et al., 1985; Mujika, de Txabarri, Maldonado-Martin, Pyne, 2012), or increased time to exhaus-tion (Ng, Cheng, Fung, Ngai, Wong, Yeung, 2007). Physiologically, the improved performance correlated to warm up may be related to the increased muscle temperature (Bishop, 2003a; Jones, Koppo, Burnley, 2003), improved $\mathrm{VO}_{2}$ kinetics early in the exercise bout (Burnley, Jones, Carter, Doust, 2000; Gerbino et al., 1996; Hajoglou et al., 2005; Johnson et al., 2014), improved exercise tolerance (Carter et al., 2005), improved aerobic abilities (Bishop, 2003a; Carter et al., 2005; Hajoglou et al., 2005; Johnson et al., 2014; Jones et al., 2003), and increased nerve conduction velocity (Johnson et al., 2014). It is hypothesized that the increase in $\mathrm{VO}_{2}$ and increase in $\mathrm{HR}$ (Bearden, Moffatt, 2001) would likely decrease the oxygen deficit and increase cardiac output, allowing for greater performance at the onset of ex-ercise. Increased cardiac output would also assist with removal of any lactic acid accu-mulated during the warm-up, resulting in fewer disturbances in blood $\mathrm{pH}$.

These positive changes have been seen with various warm-up methods includ-ing different intensities and durations. For example, increasing the intensity of a warm-up by including sprints, has been shown to be effective in long-term and intermediate performance (Grodjinovsky, Magel, 1970; Hajoglou et al., 2005). Whereas, an active low-intensity warm-up at $40 \% \mathrm{VO}_{2}$ max demonstrated a greater efficiency of thermoregu-lation in cross-country skiers, resulting in an early sweat response (Chwalbinska-Moneta, Hanninen, 1989). Johnson et al. (2014) found that an active full body warm-up resulted in an improved endurance performance during a $10 \mathrm{~K}$ cycling time trial, which supported a study by Hajoglou et al. (2005). In these cases, a warm-up was shown to improve exercise performance over no warm-up, and most of the improvements were seen during the first few minutes of the exercise bout, which researchers believed were likely due to accelerated $\mathrm{VO}_{2}$ kinetics (Hajoglou et al., 2005). In addition, cyclists that completed a low-intensity rowing warm-up of 30 minutes produced a higher mean power output compared to those that completed a 60-minute warm-up with various in-tensities (Mujika et al., 2012). Specifically, the higher power output was more promi-nent during the first 7.5 minutes of the 10-minute time trial. This study supports the no-tion that a warm-up should not be too long, as it may result in too much physiological strain.

Intensity and duration of the warm-up should be monitored to avoid significant disruptions in homeostasis such as muscle fatigue, high core temperatures, lactate production, and substrate depletion (Andzel, Busuttil, 1982; Bergstrom, Hermansen, Hultman, Saltin, 1967; Carter et al., 2005; Genovely, Stamford, 1982; Gregson et al., 2002; Kozlowski et al., 1985; Wittekind, Beneke, 2009). Warm-up intensities above lac-tate threshold tend to increase blood lactate response more than low-intensity warm-ups (Genovely, Stamford, 1982; Gray, Nimmo, 2001). These warm-ups have also been shown to hinder work output (Genovely, Stamford, 1982; Tomaras, Maclntosh, 2011), have no effect on exercise time to exhaustion (Wittekind, Beneke, 2009), or improve $\mathrm{VO}_{2}$ kinetics and muscle 
perfusion (Gerbino et al., 1996). In instances of prolonged warm-ups performed at high intensity, a decline in performance is believed to be due to glycogen depletion. However, when participants completed a prolonged warmup at an intensity below anaerobic threshold, body temperature was increased and there was no change in lactate accumulation or maximal performance (Genovely, Stamford, 1982).

Manipulation of the warm-up protocols may include alterations in the warm-up duration, intensity, or transition period. Various studies have demonstrated mixed ef-fects of a warm-up on performance due to employing different warm-up routines (inten-sity and duration) that make it difficult for uniform comparison. Furthermore, there is little evidence indicating the ideal duration and intensity of a warm-up and its effect on long-term aerobic performance. Therefore, the purpose of this study was to determine if differing durations of a warm-up completed below lactate threshold affects power out-put, $\mathrm{VO}_{2}$, and lactate during a $5 \mathrm{~K}$ cycling time trial.

\section{Material and methods}

Sixteen (males, $n=10$; females, $n=6$ ) trained cyclists ( $41.0 \pm 7.7$ years, $76.5 \pm 13.5 \mathrm{~kg}, 1.75 \pm 0.11 \mathrm{~m}, 16.3 \pm 5.8 \%$ body fat, and $50.5 \pm 11.7 \mathrm{ml} \cdot \mathrm{kg}^{-1} \cdot \mathrm{min}^{-1}$ ) volunteered to participate in the study. Trained cyclists were defined as cycling at least twice a week for the last four months. All subjects were considered low risk as defined by American College of Sports Medicine (ACSM). Exclusionary criteria included: moderate- or high-risk for cardiovascular disease as defined by the ACSM, individuals taking androgenic medications or ergogenic levels of nutritional supplements that could affect muscle mass or anabolic/catabolic hormone levels within one month prior to the start of the study. Prior to beginning the study, participants read and completed university-approved written informed consent document. All testing procedures were conducted at the Health and Human Performance Laboratory at Meredith College.

At baseline testing, participants were measured for height, weight, and body composition using the sevensite skinfold technique. The participants completed a graded exercise test for determination of $\mathrm{VO}_{2}$ max and lactate threshold. All testing was completed using a stationary cycle simulator (Computrainer, RacerMate, Seattle, WA) and the participants provided their own bicycle. The baseline testing protocol consisted of a three-minute warm-up at $25 \mathrm{~W}$, followed by gradual increases in workload over three-minute increments until the participant could no longer maintain the pace. During the test, expired respiratory gases were collected and analyzed for oxygen and carbon dioxide concentration (Mini CPX, VacuMed, Ventura, CA). A 25-microliter (1-drop) blood sample was collected from an earlobe puncture at the end of each three-minute stage and analyzed for lactate concentration (Lactate Pro, Arkray, Inc., Kyoto, Japan) to de-termine lactate threshold. HR (Polar Electro, Finland) and blood pressure were moni-tored at the end of each stage. After completion of the GXT, the participant completed a five-minute cool down followed by a familiarization with the $5 \mathrm{~K}$ time trial (TT).

This study was a randomized cross-over design, with each participant complet-ing all three testing conditions. Following baseline testing, participants completed each of the following warm-up protocols in random order one week apart: Short warm-up of three minutes at $60 \% \mathrm{VO}_{2} \max (\mathrm{SW})$, long warm-up of ten minutes at $60 \% \mathrm{VO}_{2} \max$ (LW), or no warm-up (NW). After the warm-up, participants began a $5 \mathrm{~K}$ TT at a simulated $3 \%$ grade. There was one minute between completion of the warm-up and initiation of the TT. This short time period was necessary to configure the cycle simulator to the proper settings for the TT. Participants were not privy to any performance measures except dis-tance during the TT. No verbal encouragement was provided to participants during the experimental sessions. During each trial, expired gasses and ventilation were meas-ured throughout the warm-up and the TT. HR, blood pressure, rating of perceived exer-tion (RPE), and lactate concentration were measured 
prior to the warm-up, prior to the TT, and after completion of the TT. $\mathrm{VO}_{2}$ and $\mathrm{HR}$ were also measured at the end of the first kilometer. Performance time, absolute power, relative power, cadence, and speed were obtained from the Computrainer at the $1 \mathrm{~K}$ split and at the end of the TT.

Data were analyzed with a multivariate ANOVA to address differences among type of warm-up at the completion of the TT and after completion of the first kilometer. Tukey HSD was used for post-hoc analyses. The variables that were tested included absolute power, relative power, cadence, speed, $\mathrm{HR}, \mathrm{VO}_{2}$ and lactate. An alpha level of 0.05 was used for all analyses. SPSS Statistics 19 (IBM Corporation, Somers, NY) was used for all statistical analyses.

\section{Resullts}

There was no statistical difference between types of warm-up for any of the per-formance variables, including: completion time $\left(F_{2.45}=0.231, p=0.795\right)$, absolute power $\left(F_{2.45}=0.088, p=0.916\right)$, relative power $\left(F_{2.45}=0.105\right.$, $p=0.900)$, cadence $\left(F_{2.45}=0.607, p=0.549\right)$, speed $\left(F_{2.45}=0.226, p=0.798\right)$, heart rate $\left(F_{2.45}=0.269, p=0.766\right)$, lactate $\left(F_{2.45}=0.556, p=0.577\right)$, and $V_{2}\left(F_{2.45}=0.029, p=0.971\right)$ at the completion of the $5 \mathrm{~K}$ TT. There was also no significant difference between the warm-up types for any of these variables at the end of the first kilometer (completion time: $F_{2.45}=0.928, p=0.403$; absolute power: $F_{2.45}=0.501, p=0.609$; relative power: $F_{2.45}=0.605$, $p=0.509$; cadence; $F_{2.45}=0.250, p=780$; speed: $F_{2.45}=0.952, p=0.394$; heart rate: $F_{2.45}=1.99, p=0.149 ;$ and $\mathrm{VO}_{2}: \mathrm{F}_{2.45}=2.34, \mathrm{p}=0.121$ ).

Figure 1 shows the completion time for both the $1 \mathrm{~K}$ split and the TT completion. While there was no significant difference between type of warm-up in the $1 \mathrm{~K}$ split, the NW trial was on average 10-11 s slower than the other two warm-up conditions. Fur-ther, the NW trial completion time was on average 28 seconds and 34 seconds slower than the SW and LW trials respectively.

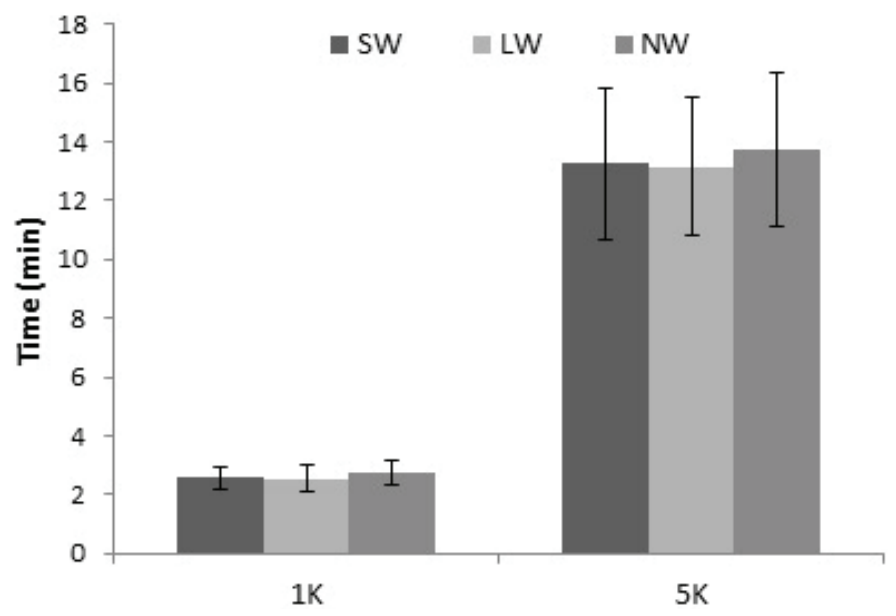

Figure 1. Mean times for the first kilometer (1K) and at the end of the $5 \mathrm{~K}$ TT. NW repre-sents the no warm-up condition, SW represents the short warm-up condition, and LW represents the long warm-up condition 
Figure 2 shows the mean $\mathrm{VO}_{2}$ during the TT for each warm-up condition. Again, there was no significant difference, but the NW condition resulted in a lower $\mathrm{VO}_{2}$ at the $1 \mathrm{~K}$ split compared to the $\mathrm{SW}$ and $\mathrm{LW}$ trials. This difference was not present at the com-pletion of the TT. The LW trial resulted in the highest $\mathrm{VO}_{2}$ at the completion of the first kilometer.

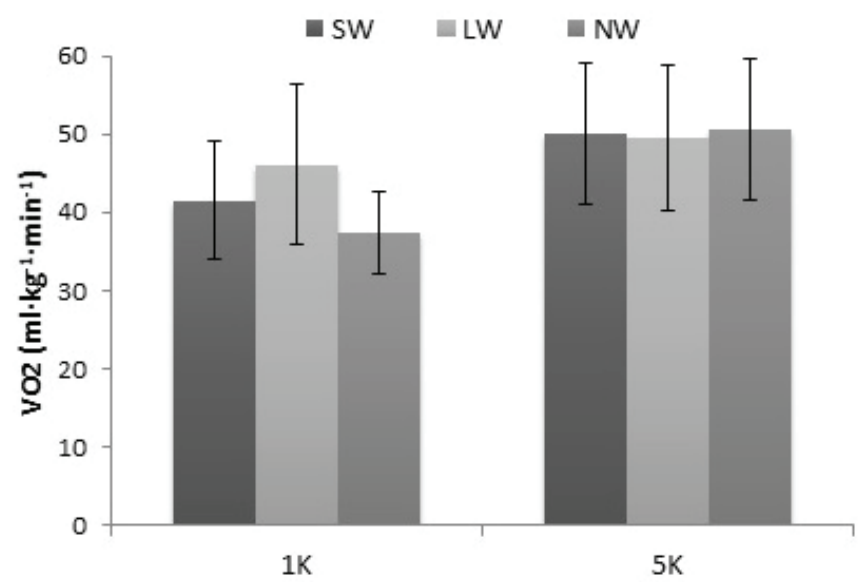

Figure 2. Mean $\mathrm{VO}_{2}$ for the first kilometer (1K) and at the end of the $5 \mathrm{~K}$ TT. NW repre-sents the no warm-up condition, SW represents the short warm-up condition, and LW represents the long warm-up condition

Figure 3 shows the differences in HR between the three warm-up conditions at the $1 \mathrm{~K}$ split and at the completion of the TT. While there was no significant difference, HR in the NW condition was slightly lower at the $1 \mathrm{~K}$ split by a mean of $8 \mathrm{bpm}$ and $11 \mathrm{bpm}$ compared to the SW and LW trials respectively. This differential was less at the completion of the TT, with a mean difference of $3 \mathrm{bpm}$ compared to the SW trial, and $4 \mathrm{bpm}$ compared to the LW trial.

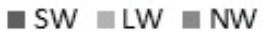

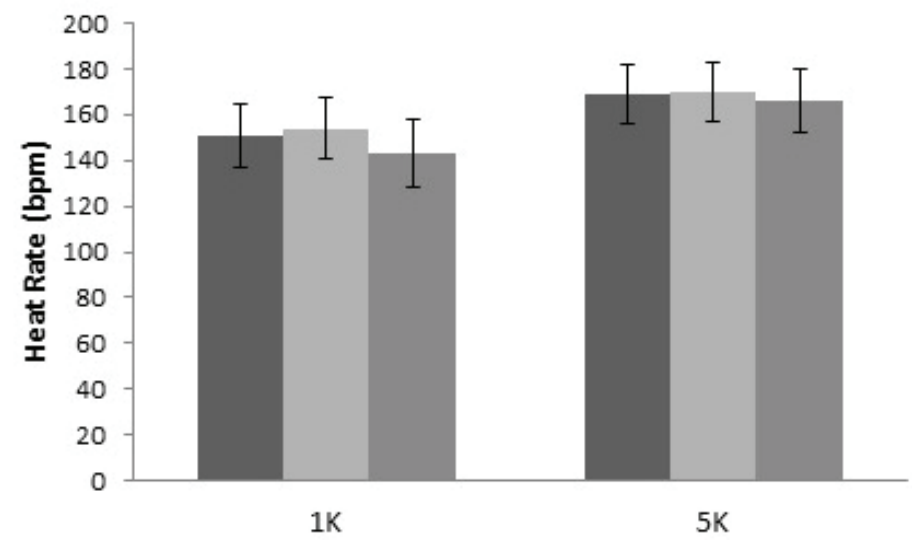

Figure 3. Mean heart rates for the first kilometer $(1 \mathrm{~K})$ and at the end of the 5K TT. NW represents the no warm-up condition, SW represents the short warm-up condition, and LW represents the long warm-up condition 
Table 1 shows all other performance-based parameters for both the first kilometer (1K) and completion of the TT. There was no statistically significant difference between the three warm-up conditions for any of these performance variables. Notably, the dif-ference between the NW trial compared to the SW and LW trials are greater at the $1 \mathrm{~K}$ split than at the completion of the TT. In each variable, the NW trial had less favorable numbers than the other two warm-up trials at the $1 \mathrm{~K}$ split. Lastly, there was also no dif-ference between type of warm-up for RPE prior to the onset of the TT (NW: N/A, SW: $10.3 \pm 1.2$, LW: $10.3 \pm 1.3$ ) or after TT completion (NW: $17.1 \pm 2.2$, SW: 17.9 \pm 1.7, LW: $17.0 \pm 1.3$ ).

Table 1. Comparison amongst type of warm-up for absolute power, relative power, ca-dence, and speed at the end of the first kilometer $(1 \mathrm{~K})$ and at the end of the $5 \mathrm{~K}$ TT. Blood lactate values are also shown at the end of the $5 \mathrm{~K}$ time trial. NW represents the no warm-up condition, SW represents the short warm-up condition, and LW represent the long warm-up condition

\begin{tabular}{lcccccc} 
& \multicolumn{3}{c}{ 1K } & \multicolumn{3}{c}{$5 \mathrm{~K}$} \\
\cline { 2 - 7 } & NW & SW & LW & NW & SW & LW \\
\hline Absolute power $(\mathrm{W})$ & $221.9 \pm 51.6$ & $235.7 \pm 60.4$ & $242.9 \pm 68.2$ & $218.8 \pm 54.4$ & $226.8 \pm 58.4$ & $225.1 \pm 57.3$ \\
Relative power $(\mathrm{W} / \mathrm{kg})$ & $2.92 \pm 0.56$ & $3.08 \pm 0.59$ & $3.19 \pm 0.77$ & $2.88 \pm 0.61$ & $2.98 \pm 0.65$ & $2.96 \pm 0.64$ \\
Cadence $(\mathrm{rpm})$ & $84.3 \pm 11.5$ & $86.6 \pm 9.8$ & $86.4 \pm 10.6$ & $90.1 \pm 8.4$ & $89.8 \pm 8.84$ & $89.6 \pm 8.74$ \\
Speed $(\mathrm{km} / \mathrm{h})$ & $22.5 \pm 3.9$ & $23.3 \pm 4.0$ & $23.3 \pm 3.7$ & $27.1 \pm 3.5$ & $27.3 \pm 4.1$ & $28.7 \pm 5.4$ \\
Lactate $(\mathrm{mmol} / \mathrm{l})$ & - & - & - & $8.0 \pm 3.2$ & $9.0 \pm 2.9$ & $7.9 \pm 3.2$ \\
\hline
\end{tabular}

\section{Discussion}

The results indicate that warm-up duration had no impact on performance time, $\mathrm{VO}_{2}$, power output, $\mathrm{HR}$, cadence, speed, or RPE at completion of the $5 \mathrm{~K}$ TT or in the initial $1 \mathrm{~K}$. There was also no significant difference among types of warm-up for blood lactate accumulation at the end of the 5K TT. These results agree with previous studies that have demonstrated no improvement in performance when having completed a warm-up with no rest prior to long duration exercise performance (Andzel, 1978; Andzel, Gutin, 1976; Grodjinovsky, Magel, 1970), and with studies that employed a small rest period after the warm-up period (Gray, Nimmo, 2001; Gregson et al., 2002; Wittekind, Beneke, 2009).

Andzel and Gutin (1976) researched the effect of a short rest period, 30 or 60 seconds, between a warmup and a bench stepping task. The study found that this rest period improved performance when compared to subjects who completed no warm-up or no rest between the warm-up and the step task. In a later study by Andzel (1978), the effects of no warm-up and varied rest times between warm-up, and a treadmill task were compared to no warm-up before the task. The rest periods of $30,60,90$, and 120 sec-onds were tested, and the rest period of 30 seconds yielded the best performance. The authors suggested that the rest period might have been beneficial in allowing any lac-tate formed during the warm-up to be cleared out, as well as complete at least partial resynthesis of phosphagens. However, in the current study, the warm-up protocol was below that of anaerobic threshold, which would likely suggest that lactate accumulation would not have been a contributor to the exercise performance. Because lactate likely did not accumulate it would not have affected the overall $5 \mathrm{~K}$ or the $1 \mathrm{~K}$ split performanc-es.

Studies that showed a warm-up improved performance attributed the benefit to acceleration of $\mathrm{VO}_{2}$ kinetics (Hajoglou et al., 2005), an increase in anaerobic threshold (Chwalbinska-Moneta, Hanninen, 1989), and structure of the warm-up (Johnson et al., 2014). Hajoglou et al. (2005) and Carter et al. (2005) both used varying intensities of warm-up prior to exercise, incorporating times of higher and lower intensities within the same warm-up. Hajaglou et al. found that performance was improved despite the inten-sity of warm-up. Carter et al. found that a heavy warm- 
up, three 73 -second cycling ef-forts at $90 \%$ critical power, lengthened total time to exhaustion by $10 \%$ while a severe warm-up, three 60 -second cycling efforts at $110 \%$ critical power, hindered the subse-quent exercise performance. In the current study, the data at $1 \mathrm{~K}$ into the TT, while not statistically significant, indicate a small benefit in warm-up exercise prior to perfor-mance with faster times, higher power outputs, higher cadences, and higher $\mathrm{VO}_{2}$ for both of the warm-up trials compared to the NW trial. The mean $1 \mathrm{~K}$ time differential be-tween NW and either type of warm-up was approximately 10 seconds. While this was not substantial for the recreationally trained population utilized in this study, this would likely be substantial for a more competitive or elite level population. Further, when ad-dressing the finishing time for the $5 \mathrm{~K}$ TT, the differences between the NW trial and the SW and LW trials were approximately $28 \mathrm{~s}$ and $33 \mathrm{~s}$ respectively, with the NW trial per-forming worse in each case. This differential would probably decrease over an extend-ed TT range like those used in professional cycling races, but would still be impactful regarding the overall standings in the race. For recreational individuals that embark on a training ride, a warm-up isn't going to significantly impact the workout and is not nec-essary. It should also be noted that this study was conducted in a thermoneutral envi-ronment, the impact of a warm-up for recreationally trained cyclists may be different when exercising in cooler or warmer temperatures.

Following completion of the study, participants were asked which trials they felt were their fastest and slowest times, and which trial they felt was the most difficult. The consensus from the participants was that the NW trial was much more difficult at the onset of exercise compared to the other trials, and they thought it was their slowest time. Ng, Cheng, Fung, Ngai, Wong, Yeung (2007) studied the effects of a warm-up in time to reach a specific rate of perceived exertion (RPE) using a cycling protocol. Sub-jects cycled under a constant power output until they felt they had reached an RPE of 15 . The study found that the passive warm-up had significantly longer time to RPE than compared to the control of no warm-up and active warm-up. However, another study that compared subject's RPE to varying warm-up protocols found that both a cycling warm-up and a cycling and inspiratory muscle warm-up elicited higher RPE responses than the control of no warm-up (Johnson et al., 2014). These authors attributed the higher RPE responses from a warm-up to an increased motivation and effort in the subject that was caused by the active warm-up. Some indi-viduals use the warm-up time to help prepare them mentally to concentrate on the upcoming event. In the absence of a warm-up, this psychological preparation time is lost, and this may have an impact on performance (Bishop, 2003b).

Coaches, athletes, and personal trainers regularly emphasize the importance of completing a quality warmup prior to aerobic exercise participation or athletic perfor-mance citing improved $\mathrm{VO}_{2}$ kinetics, improved muscular thermal environment, en-hanced muscular blood flow, and improved cardiovascular system functioning. While these physiological changes are important to how one feels early during exercise by reducing oxygen deficit, they likely did not translate to significantly improved perfor-mance during the exercise bout. Thus, it may be unnecessary for recreational athletes performing long duration exercise to perform a lengthy structured warm-up prior to the exercise bout. However, coaches and athletes should still consider the use of a warm-up prior to aerobic exercise because the small difference seen during the onset of ex-ercise may affect the overall performance of the athlete while in competition. Additional-ly, including a warm-up prior to daily training could also help athletes establish a rou-tine, thus enhancing mental and physical preparedness prior to competition. 


\section{Conclusions}

To our knowledge, this is the first study that measured the effects of active warm-up duration below lactate threshold on performance in a competitive cycling time trial. Although no significant differences between types of warm-up were demonstrated at completion of the time trial, these findings are important as there were no significant differences after the first kilometer of the time trial. This indicates that the potential ef-fects of having completed a warm-up (e.g. enhanced $\mathrm{VO}_{2}$ kinetics, increased muscle temperature, and improved muscle blood flow) were no longer present less than four minutes into the exercise activity. Additionally, completing a warm-up may be impactful on the mentality and subjective effort of the athlete.

\section{References}

Andzel, W.D. (1978). The effects of moderate prior exercise and varied rest intervals upon cardiorespiratory endurance performance. J Sports Med Phys Fitness, 18 (3), 245-252.

Andzel, W.D. (1982). One mile run performance as a function of prior exercise. J Sports Med Phys Fitness, 22 (1), 80-84.

Andzel, W.D., Busuttil, C. (1982). Metabolic and physiological responses of college females to prior exercise, varied rest intervals and a strenuous endurance task. J Sports Med Phys Fitness, 22 (1), 113-119.

Andzel, W.D., Gutin, B. (1976). Prior exercise and endurance performance: a test of the mobilization hypothesis. Res Q, 47 (2), 269-276.

Bearden, S.E., Moffatt, R.J. (2001). $\mathrm{VO}_{2}$ and heart rate kinetics in cycling: transitions from an elevated baseline. J Appl Physiol (1985), 90 (6), 2081-2087.

Bergstrom, J., Hermansen, L., Hultman, E., Saltin, B. (1967). Diet, muscle glycogen and physical performance. Acta Physiol Scand, $71(2), 140-150$.

Bishop, D. (2003a). Warm up I: potential mechanisms and the effects of passive warm up on exercise performance. Sports Med, 33 (6), 439-454.

Bishop, D. (2003b). Warm up II: performance changes following active warm up and how to structure the warm up. Sports Med, 33 (7), 483-498.

Burnley, M., Jones, A.M., Carter, H., Doust, J.H. (2000). Effects of prior heavy exercise on phase II pulmonary oxygen uptake kinetics during heavy exercise. J Appl Physiol (1985), 89 (4), 1387-1396.

Carter, H., Grice, Y., Dekerle, J., Brickley, G., Hammond, A.J., Pringle, J.S. (2005). Effect of prior exercise above and below critical power on exercise to exhaustion. Med Sci Sports Exerc, 37 (5), 775-781.

Chwalbinska-Moneta, J., Hanninen, O. (1989). Effect of active warming-up on thermoregulatory, circulatory, and metabolic responses to incremental exercise in endurance-trained athletes. Int J Sports Med, 10 (1), 25-29.

Genovely, H., Stamford, B.A. (1982). Effects of prolonged warm-up exercise above and below anaerobic threshold on maximal performance. Eur J Appl Physiol Occup Physiol, 48 (3), 323-330.

Gerbino, A., Ward, S.A., Whipp, B.J. (1996). Effects of prior exercise on pulmonary gas-exchange kinetics during high-intensity exercise in humans. J Appl Physiol (1985), 80 (1), 99-107.

Gray, S., Nimmo, M. (2001). Effects of active, passive or no warm-up on metabolism and performance during high-intensity exercise. J Sports Sci, 19 (9), 693-700.

Gregson, W.A., Drust, B., Batterham, A., Cable, N.T. (2002). The effects of pre-warming on the metabolic and thermoregulatory responses to prolonged submaximal exercise in moderate ambient temperatures. Eur J Appl Physiol, 86 (6), 526-533.

Grodjinovsky, A., Magel, J.R. (1970). Effect of warm-up on running performance. Res Q, 41 (1), 116-119.

Hajoglou, A., Foster, C., De Koning, J.J., Lucia, A., Kernozek, T.W., Porcari, J.P. (2005). Effect of warm-up on cycle time trial performance. Med Sci Sports Exerc, 37 (9), 1608-1614.

Johnson, M.A., Gregson, I.R., Mills, D.E., Gonzalez, J.T., Sharpe, G.R. (2014). Inspiratory muscle warm-up does not improve cycling time-trial performance. Eur J Appl Physiol, 114 (9), 1821-1830.

Jones, A.M., Koppo, K., Burnley, M. (2003). Effects of prior exercise on metabolic and gas exchange responses to exercise. Sports Med, 33 (13), 949-971. 
Kozlowski, S., Brzezinska, Z., Kruk, B., Kaciuba-Uscilko, H., Greenleaf, J.E., Nazar, K. (1985). Exercise hyperthermia as a factor limiting physical performance: temperature effect on muscle metabolism. J Appl Physiol (1985), 59 (3), 766-773.

Martin, B.J., Robinson, S., Wiegman, D.L., Aulick, L.H. (1975). Effect of warm-up on metabolic responses to strenuous exercise. Med Sci Sports, 7 (2), 146-149.

Mujika, I., de Txabarri, R.G., Maldonado-Martin, S., Pyne, D.B. (2012). Warm-up intensity and duration's effect on traditional rowing time-trial performance. Int J Sports Physiol Perform, 7 (2), 186-188.

Ng, G., Cheng, C., Fung, M., Ngai, N., Wong, E., Yeung, A. (2007). Comparison of the time to perceived exertion in cycling with different warm-up procedures. Hong Kong Physiotherapy Journal, 25 (1), 10-13.

Tomaras, E.K., MacIntosh, B.R. (2011). Less is more: standard warm-up causes fatigue and less warm-up permits greater cycling power output. J Appl Physiol (1985), 111 (1), 228-235.

Wittekind, A.L., Beneke, R. (2009). Effect of warm-up on run time to exhaustion. J Sci Med Sport, 12 (4), 480-484.

Cite this article aS: Bunn, J.A., Eschbach, L.C., Magal, M., Wells, E.K. (2017). The Effectsof Warm-up Duration on Cycling Time Trial Performance in Trained Cyclists. Central European Journal of Sport Sciences and Medicine, 17 (1), 5-13. DOI: 10.18276/cej.2017.1-01. 
